\title{
Primary Neuroendocrine Carcinoma of Breast
}

\author{
Khamar Jaha Banu • Rajesh Ballal C • Narendra Pai • \\ Harish Permi
}

Received: 7 February 2014 / Accepted: 17 December 2014 / Published online: 25 January 2015

(C) Indian Association of Surgical Oncology 2015

\begin{abstract}
Neuroendocrine differentiation can be identified in a subset of human breast carcinomas, either as scattered cells or as a predominant neuroendocrine component. Clinically, there are no notable or specific differences in presentation from other type of breast tumors. The diagnosis of neuroendocrine breast carcinoma relies mainly on histopathological examination and immunohistochemistry marker study. Treatment modalities are not different from those for the other conventional types. We report a case of primary neuroendocrine carcinoma of breast with relevant discussion on neuroendocrine tumor.
\end{abstract}

Keywords Primary neuroendocrine carcinoma $\cdot$ Breast . Immuno histochemistry

\section{Introduction}

Neuroendocrine breast carcinomas (NEBC) are one of the rarest tumours with only about 30 cases been reported in the literature [1]. Neuroendocrine carcinomas are slow-growing tumors derived from neuroendocrine cells, which are present throughout the body. They arise most commonly in the bronchopulmonary system and gastrointestinal tract. NEBC's are rare tumors accounting for less than $0.1 \%$ of all breast cancer [1]. History and clinical examination do not play a

\footnotetext{
K. J. Banu $\cdot$ R. Ballal C $\cdot$ N. Pai $(\bowtie)$

Department of General Surgery, K S Hegde Hospital,

Deralakate, Mangalore 575018, India

e-mail: drnaren_86@yahoo.com

H. Permi

Department of Pathology, K S Hegde Hospital,

Deralakate, Mangalore 575018, India
}

significant role in diagnosing NEBC as most of the patients present with common symptoms like painless lump. Although Fine needle aspiration cytology and histopathology might reveal neuroendocrine elements, positive neuroendocrine markers [neuron specific enolase, chromogranin, synaptophysin and cytokeratin] will give strong support to the diagnosis. In Immunohistochemistry (IHC) marker study, if the tumour population is positive for more than $50 \%$ then the diagnosis of primary NEBC has to be considered. The prognosis is still a matter of debate; however, when compared with unselected breast cancers, it shows a less aggressive clinical behavior.

We report a case of Primary NEBC in a 54 year old female presenting with breast lump, which was diagnosed by histopathology and confirmed by immunohistochemistry markers study.

\section{Case Report}

A 54 year old female presented with painless lump in the right breast since 5 months. Local examination showed a solitary immobile lump in upper outer quadrant measuring $3 \times 4 \mathrm{cms}$ which was firm in consistency. Mammography was suggestive of malignancy (BIRADS-5). Fine Needle Aspiration Cytology(FNAC) of the lump showed features suggestive of infiltrating duct carcinoma breast. She underwent Modified Radical Mastectomy(MRM) and specimen was sent for histopathological examination.

Grossly breast specimen measured $20 \times 10 \times 4.5 \mathrm{cms}$ with elliptical piece of skin showing nipple and areola. Cut section revealed an ill-defined greywhite growth measuring $2.4 \times$ $1.6 \mathrm{cms}$ (Fig. 1). Microscopy showed tumor tissue arranged in solid sheets and nesting pattern separated by fibrovascular stroma. The tumor cells were small and uniform containing moderate amount of granular eosinophilic cytoplasm, 


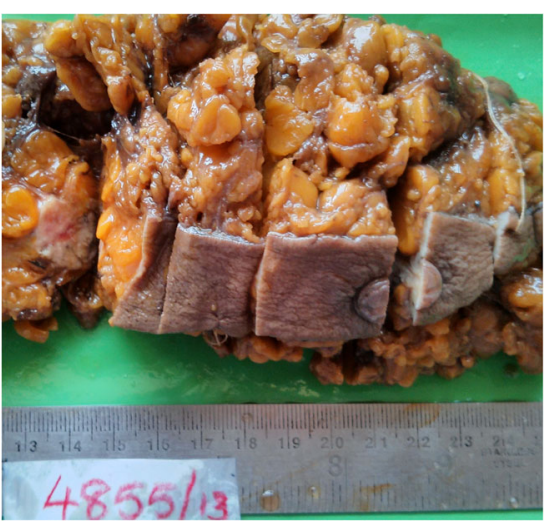

Fig. 1 Grossly the specimen measured $20 \times 10 \times 4.5 \mathrm{cms}$ with the growth measuring $2.4 \times 1.6 \mathrm{cms}$ with areas of haemorrhage

enlarged vesicular nucleus with stippled chromatin (Fig. 2). Histopathology was suggestive of infiltrating ductal carcinoma breast with neuroendocrine differentiation. Immunohistochemistry marker study showed $50-55 \%$ and $55-60 \%$ positivity of tumor cells to chromogranin A (Fig. 3) and Neuron specific enolase (Fig. 4) respectively. Tumour cells were also diffusely and strongly positive for cytokeratin (Fig. 5), estrogen, and progesterone receptors and negative for Her-2-neu receptor. Two lymph nodes showed features of reactive lymphadenitis. Modified Scarff Bloom Richardson Grading was Grade II. TNM pathological staging was $\mathrm{pT}_{2} \mathrm{~N}_{\mathrm{x}} \mathrm{M}_{\mathrm{X}}$-Stage II. CT scan of thorax and abdomen was normal. Final diagnosis of Primary neuroendocrine breast carcinoma was made. Post-operatively, 6 cycles of adjuvant chemotherapy was given and tablet tamoxifen was started after chemotherapy. Patient was followed up every six monthly for first year. Mammogram of opposite breast, ultrasound abdomen, chest X-Ray was done 1 year after surgery which was normal. Hereafter she is advised follow up once in 6 month for one more year and thereafter once in a year for next 3 years.

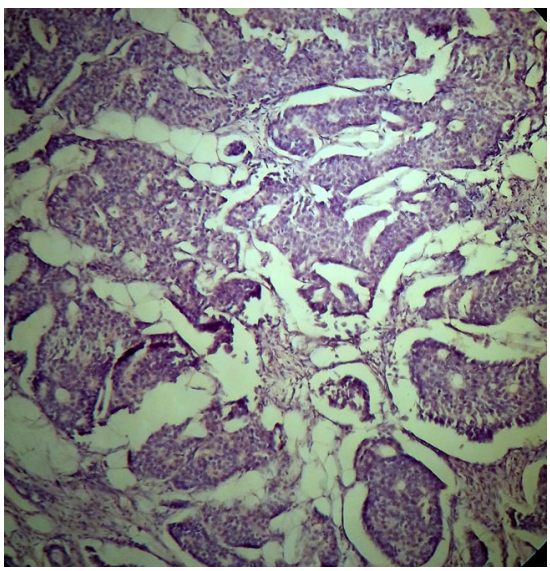

Fig. 2 On light microscopy(H and E) the tumour showed cribriform, solid and papilarry pattern. The tumor cells were arranged in solid sheets, nests separated by fibrovascular stroma. The cells were relatively small and uniform in size with moderate amount of granular eosinophilic cytoplasm,enlarged nucleus with strippled chromatin

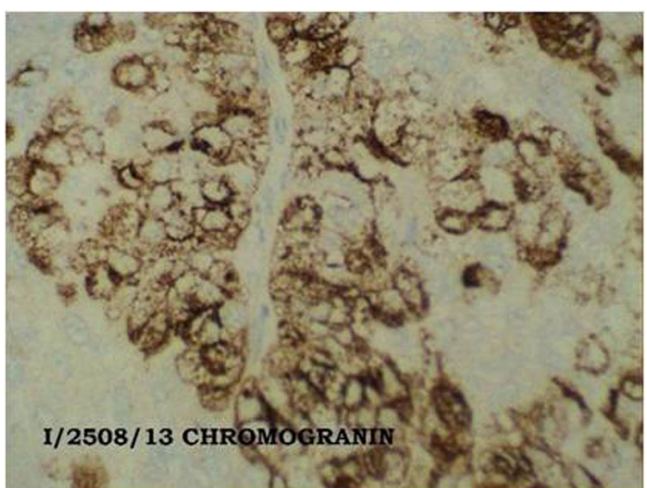

Fig. 3 IHC- $50-55 \% \%$ of tumour cells shows strong cytoplasmic reactivity to chromogranin

\section{Discussion}

Neuroendocrine breast carcinoma was originally described in 1963 by Feyrter. They identified several cases of invasive breast cancer with carcinoid growth pattern [2]. Neuroendocrine tumors are derived from neuroendocrine cells and the most common sites are the bronchopulmonary system followed by gastrointestinal tract. Breast lacks endocrine cells therefore theoretically, primary neuroendocrine tumors do not occur in the breast. It has been hypothesized that neuroendocrine breast carcinomas do not arise from pre-existing endocrine cells, but are rather the result of the differentiation process within breast carcinoma [3].

Primary NEBC are rare tumors comprising of less than $0.1 \%$ of all breast cancers [1]. The reported age incidence ranges from 20 to 83 years with a higher incidence occurring in patients aged more than 50 years. Only two cases were reported in men [4]. Clinically it is difficult to differentiate between NEBC from other tumours of the breast as patients present only with a lump and no other significant symptoms. Axillary lymphadenopathy is not very common [4].

NEBC on microscopic examination show solid sheets and nests of tumor cells separated by fibrovascular stroma. Focal neuroendocrine differentiation can be found in different histological subtypes of breast carcinoma including in situ and

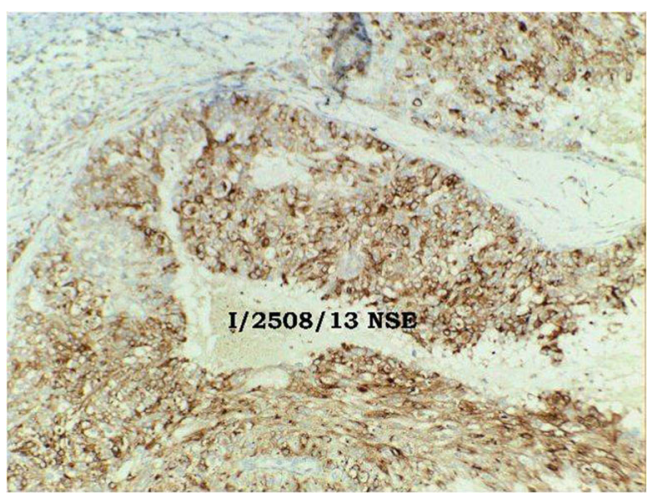

Fig. 4 IHC- $55-60 \%$ of tumour cells show strong cytoplasmic reactivity to NSE 


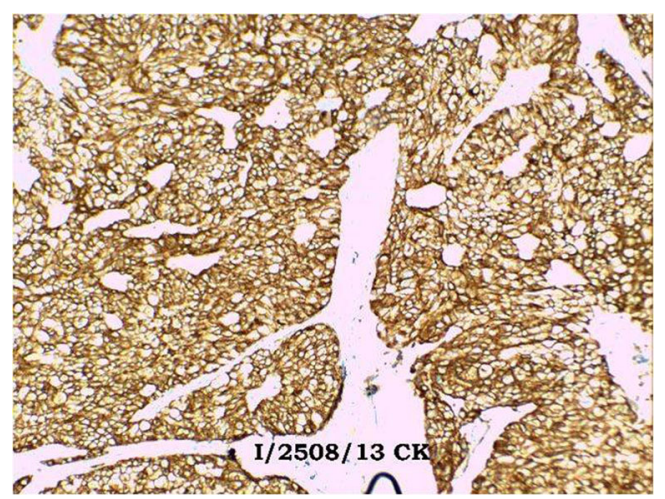

Fig. 5 IHC- Tumour diffusely and strongly positive to cytokeratin

invasive ductal, lobular, colloid or papillary breast cancer. The term neuroendocrine carcinoma is applied when more than $50 \%$ of tumor shows such differentiation [4].

In literature there are different classifications, according to the specific features of histopathology that have been proposed for neuroendocrine tumors, arising within the breast. Amongst these the most realistic is the WHO(World Health Organisation) classification, which defines three principle tumors, i.e., solid neuroendocrine carcinoma, small cell/oat cell carcinoma, and large cell neuroendocrine carcinoma [5]. It should be noted, however, that not all breast tumors that exhibit neuroendocrine features completely fit into the WHO classification categories [6]. Our case falls under the category of solid neuroendocrine carcinoma.

Histopathological examination in our case revealed the presence of neuroendocrine differentiation because of the existence of nest and solid structures of group of uniform cells with round to oval vesicular nuclei with stippled chromatin and granular eosinophilic cytoplasm.

The neuroendocrine markers neuron-specific enolase (55$60 \%$ ) and Chromogrannin-A (50-55\%) should be positive in the tumor cells, to consider the diagnosis of primary neuroendocrine breast tumor [7] which was seen in our case.

While making the diagnosis of neuroendocrine carcinoma of breast and prior to the management, it is imperative to classify these tumours as 'primary' or 'metastatic' in origin [8]. The surgeon should focus on ruling out breast metastasis from small cell carcinoma of the lung, the gastrointestinal tract, pancreas, and cervix. Metastatic neuroendocrine carcinoma from other organs cannot be distinguished from primary because histologically they are similar [9]. The role of imaging plays a vital role in distinguishing between primary and metastatic NEBC. A contrast enhanced CT scan of the thorax and abdomen was done in order to rule out other primary sites.

Treatment modalities for NEBC are not different from those available for the other conventional types of breast carcinoma. Currently specific recommendations regarding surgical management do not exist though it has been advocated that the choice of surgical procedure depends on the tumor's location and clinical stage. There is limited information regarding safety of breast conservation surgery (BCS) and immediate breast reconstruction.

Currently there is no information that indicates the most efficacious chemotherapeutic regimen [4]. The general protocol is to treat it with chemotherapy regimens as followed in other conventional types of breast cancers. In this current case patient underwent MRM followed by a chemotherapeutic regimen of cyclophosphamide, adriamycin and 5-Flourouracil(5FU). There is a limited role of radiation therapy in the treatment of NEBC. The recommendation for hormonal therapy is based on their hormonal receptor positivity [4].The prognostic significance cannot be commented upon due to only a small number of cases being reported till date.

\section{Conclusion}

To conclude, primary neuroendocrine carcinoma of the breast is a group that exhibits morphological features that resemble classic neuroendocrine tumors. The definitive diagnosis can only be made with help of histopathology and immunohistochemical markers. This case of primary neuroendocrine breast carcinoma is presented because of its rarity.

As of now, the treatment modalities are not different from those for the other conventional types. Currently it is difficult to fully understand this rare tumor because issues such as histogenesis, optimal adjuvant therapy, and prognosis are still unknown as only a small number of cases have been reported till date.

\section{References}

1. Singh S, Aggarwal G, Kataria SP, Kalra R, Duhan A, Sen R (2011) Primary neuroendocrine carcinoma of breast. J Cytol 28(2):91-92

2. Feyrter F, Harmann G (1963) On the carcinoid growth form of the carcinoma mammae, especially the carcinoma solidum (gelatinosum) mammae. Frankf Z Pathol 73:24-39

3. Maluf HM, Koerner FC (1994) Carcinomas of the breast with endocrine differentiation: a review. Virchows Arch 425(5):449-457

4. Angarita FA, Rodríguez JL, Eugenio M, Sánchez JO, Tawil M, Torregrosa L (2013) Locally-advanced primary neuroendocrine carcinoma of the breast: case report and review of the literature. World $\mathrm{J}$ Surg Oncol 5:11-128

5. Chang ED, Kim MK, Kim JS, Whang IY (2013) Primary neuroendocrine tumor of the breast: imaging features. Korean J Radiol 14(3):395-399

6. Tavassoli FA, Devilee P (2003) Tumours of the breast. In: Tavassoli FA, Devilee P (eds) Pathology and genetics of tumours of the breast and female genital organs, World Health Organization Classification of Tumours Series. IARC Press, Lyon, pp 32-34

7. Adegbola T, Connolly CE, Mortimer G (2005) Small cell neuroendocrine carcinoma of the breast: a report of three cases and review of the literature. J Clin Pathol 58:775-778

8. Tajima S, Horiuchi H (2013) Neuroendocrine tumor, well differentiated, of the breast: a relatively high-grade case in the histological subtype. Case Rep Pathol. doi:10.1155/2013/204065

9. Akhtar K, Zaheer S, Ahmad SS, Hassan MJ (2009) Primary neuroendocrine carcinoma of the breast. Indian J Pathol Microbiol 52(1):71-3 